\title{
Bioaccumulation d'aluminium chez la truite Salmo truffa fario soumise au retombées des pluies acides : étude structurale, ultrastructurale et microanalytique Aluminium bioaccumulation in the brown trout Salmo truffa fario from acidified streams: a structural, ultrastructural and microanalytical study
}

\author{
C. Chassard-Bouchaud, Ch. Galle et E. Lopez-Rabereau
}

Volume 5, numéro 1, 1992

URI : https://id.erudit.org/iderudit/705119ar

DOI : https://doi.org/10.7202/705119ar

\section{Aller au sommaire du numéro}

\section{Éditeur(s)}

Université du Québec - INRS-Eau, Terre et Environnement (INRS-ETE)

\section{ISSN}

0992-7158 (imprimé)

1718-8598 (numérique)

\section{Découvrir la revue}

Citer cet article

Chassard-Bouchaud, C., Galle, C. \& Lopez-Rabereau, E. (1992). Bioaccumulation d'aluminium chez la truite Salmo truffa fario soumise au retombées des pluies acides : étude structurale, ultrastructurale et microanalytique. Revue des sciences de l'eau / Journal of Water Science, 5(1), 37-54. https://doi.org/10.7202/705119ar

\section{Résumé de l'article}

Des études microanalytiques ont été menées sur des truites Salmo trutta fario, âgées de deux ans, récoltées dans une rivière des Vosges soumise aux retombées des pluies acides et sur des truites témoins récoltées dans une rivière d'Auvergne, non soumise aux pluies acides. La rivière des Vosges est caractérisée par un pH de 5,42 et par une concentration en aluminium de 200 $\mu \mathrm{g} / \mathrm{L}-1$. Notre but étant de déterminer les tissus, cellules et organites cibles de bioaccumulation éventuelle de l'aluminium, nous avons analysé rein, foie, branchie et tractus digestif. Deux méthodes microanalytiques ont été utilisées pour localiser l'aluminium à l'échelle cellulaire et subcellulaire et connaître les éléments avec lesquels il peut être associé; ce sont la spectrométrie de masse par émission ionique secondaire (microscope ionique associé à un système informatisé de traitement d'images) et la spectrométrie des rayons $\mathrm{X}$ (microsonde électronique de Castaing associée à un microscope électronique à transmission).

La microanalyse des rein, foie, branchie et tractus digestif montre l'existence de deux processus conduisant à la bioaccumulation de l'aluminium. Le premier, classiquement connu pour d'autres métaux, met en évidence une insolubilisation de l'aluminium sous forme de phosphate, dans des organites limités par une membrane : les lysosomes et les granules pigmentaires des mélanocytes. Le second, démontre la formation de volumineux dépots extra-cellulaires, atteignant $100 \mu \mathrm{m}$ de long et entraînant la destruction du tissu. Aucune bioaccumulation significative d'aluminium n'a été observée chez des truites témoins, récoltées dans le centre de la France, où l'eau à pH 7.9 est dépourvue d'aluminium. 


\title{
Bioaccumulation d'aluminium chez la truite Salmo trutta fario soumise au retombées des pluies acides : étude structurale, ultrastructurale et microanalytique*
}

\author{
Aluminium bioaccumulation in the brown trout \\ Salmo trutta fario from acidified streams : \\ a structural, ultrastructural and microanalytical study
}

\section{CHASSARD-BOUCHAUD ${ }^{\dagger}$, Ch. GALLE², E. LOPEZ-RABEREAU3}

Reçu le 29 octobre 1990, accepté pour publication le 10 septembre $1991^{* *}$.

\section{SUMMARY}

The major harm caused by acidic precipitation is shown by a disappearance of fish. Other factors besides acidity such as aluminium levels are significantly harmful and many studies have shown that aluminium ions are toxic to fish. The only sensible course of action is to investigate the basic mechanisms by which each of the metal pollutants enters and attacks living systems. For this, one needs to be a combination ol physicist, chemist, biochemist, physiologist and toxicologist. Investigations on metal bioaccumulation require very sensitive analytical instrumentation. Total analytical methods commonly used are inadequate : absorbed and adsorbed elements cannot be distinguished.

Therefore, interesting information can be obtained by using physical methods of chemical microanalysis : two available microanalytical techniques are particularly suitable, $X$-ray spectrometry and secondary ion mass spectrometry.

$X$-ray spectrometry, also called Electron Probe X-ray Microanalysis or Electron Microprobe (EMP) provides a means for studying the local chemical composition and structure of biological specimens. EMP tan be used in association with a photon microscope or with a transmission electron microscope allowing the detection of elements at subcellular level.

The Secondary Ion Mass Spectrometry (SIMS), also ca Iled Ion Microscopy, allows to visualize, analyse and photograph the microscopical distribution

1. Laboratoire de Biologie et Physiologie des Organismes marins, Université Pierre et Marie Curie, 4, place Jus sieu, F 75252 Paris Cedex 05 et Centre de Microanalyse appliquée ł̇ la Biologie du C.N.R.S., S.C. 27 de I'I.N.S.E.R.M., Facuité de Médecine de Créteil, 6, rue du Général Sarrail, F 94000 Créteil.

2. Laboratoire d'Hydrologie - Institut Louise Blanquet - Faculté de Médecine et de Pharmacie de ClermontFerrand, 28, place Henri Dunant, F 63000 Ciemont-Ferrand Cedex.

3. Laboratoire de Physiologie générale et comparée du Muséum National d'Histoire Naturelle, CNRS URA 90, 7, rue Cuvier, 75005 Paris.

* Communication presentée au 34e Congrès de l'Association Française de Limnologie, Metz-Nancy, 29-31 mai 1990. 
of the stable or radioactive isotopes of the elements present in a histological section. The sensitivity of the method is very high, ranging from 0.1 to $1 \mathrm{ppm}$. In association with SiMS, a processing of secondary ion images is used.

Two years-old samples of Salmo trutta fario, from wild populations living in acidified waters of Eastern France (near Cornimont in the Vosges moutains) were studied for aluminium detection at cellular and subcellular levels. The acidified waters were characterized by a low $\mathrm{pH}(5,42)$ and high aluminium level reaching $200 \mu \mathrm{g} / \mathrm{L}^{-1}$. Control trouts living in non acidified waters $(\mathrm{pH}: 7.9$ and aluminium free) of central France (near Clermont-Ferrand) were used for comparison. In order to determine the tissues, cells and organelles of a possible aluminium concentration, the following organs were investigated : kidney, liver, gill and digestive tractus, using both microanalytical techniques described above.

In the kidney ion images showed aluminium emission from tubule lysosomes with a ring-shaped localization along the apical border of the epithelial cells; aluminium emissions from the tubule lumen and from the pigment granules of the melanocytes were also observed. Using the electron microprobe, $X$-ray emission spectra of aluminium associated with phosphorus were obtained from lysosomes and pigment granules. In the liver and in the gills, ion images showed a high aluminium emission from the same organelles and $X$-ray spectra of aluminium and phosphorus were also obtained. Moreover, in the pyloric caeca, large extracellular deposits of aluminium were detected : they measured about $100 \mu \mathrm{m}$ in length and were located in places where tissues had been destroyed.

The same structural, ultrastructural and microanalytical investigations were performed on the control trouts from which non aluminium detection was obtained.

In conclusion, two processes appear to be involved in the aluminium accumulation in the brown trout. The first one corresponds to a well known insolubllisation of aluminium phosphate inside the lysosomes, due to an acidic phosphatase enzymatic activity; aluminium is also trapped inside pigment granules. Both of these mechanisms of storage inside membrane-limited orga nelles, prevent cells from any interior damage. The second one corresponds to the formation of large extracellular deposits which are likely to provoke injuries leading to the tissue destruction. Such data demonstrating basic mechanisms of aluminium accumulation in a fish, could not have been obtained using total analytical methods.

Key-words : trout, acid rain, aluminium, microanalysis (ion microscopy and $X$ ray spectrometry) ultrastructure, lysosome.

Des études microanalytiques ont été menées sur des truites Salmo trutta fario, agées de deux ans, récoltées dans une rivière des Vosges soumise aux retombées des pluies acides et sur des truites témoins récoltées dans une rivière d'Auvergne, non soumise aux pluies acides. La rivière des Vosges est caractérisée par un pH de 5,42 et par une concentration en aluminium de $200 \mu \mathrm{g} / \mathrm{L}^{-1}$. Notre but étant de déterminer les tissus, cellules et organites cibles de bioaccumulation éventuelie de l'aluminium, nous avons analysé rein, foie, branchie et tractus digestif. Deux méthodes microanalytiques ont été utilisées pour localiser l'aluminitum à l'échelle cellulaire et subcellulaire et connaître les éléments avec lesquels il peut étre associé ; ce sont la spectrométrie de masse par émission ionique secondaire (microscope ionique associé à un système informatisé de traitement d'images) et la spectrométrie des rayons $X$ (microsonde électronique de Castaing associée à un microscope électronique à transmission). 
La microanalyse des rein, foie, branchie et tractus digestif montre l'existence de deux processus conduisant à la bioaccumulation de l'aluminium. Le premier, classiquement connu pour d'autres métaux, met en évidence une insolubilisation de l'aluminium sous forme de phosphate, dans des organites limités par une membrane : les lysosomes et les granules pigmentaires des mélanocytes. Le second, démontre la formation de volumineux dépots extracellulaires, atteignant $100 \mu \mathrm{m}$ de long et entrainant la destruction du tissu. Aucune bioaccumulation significative d'aluminium n'a été observée chez des truites témoins, récoltées dans le centre de la France, où l'eau à pH 7.9 est dépourvue đ'aluminium.

Mots clés: truite, pluies acides, aluminium, microanalyse (microscopie ionique, spectrométrie des rayons $X$ ), ultrastructure, lysosomes.

\section{INTRODUCTION}

L'acidification de l'environnement est causée par le rejet d'anhydride sulfureux et d'oxydes d'azote dans l'atmosphère et par l'interaction subsé. quente de ces substances avec les différents milieux. La combustion du charbon et du pétrole, ainsi que les activités industrielles qui leur sont associées, en sont les principales sources contaminant en particulier les pluies qui deviennent acides. Ces pluies sont les responsables de désastres écologiques engendrant le dépérissement des forêts et l'acidification des eaux de surface. Ces problèmes, d'intérêt mondial sont bien connus en Scandinavie (OVERREIN et al., 1981) et aux Etats-Unis notamment (BONNEAU et LANDMANN, 1988 ; MOHMEN, 1988). En France, la région la plus exposée se situe dans les Vosges où le rôle des pluies, dans l'acidification des rivières a été démontré (MASSABUAU et al., 1987).

Les deux éléments majeurs mis en cause dans la toxicité des eaux acides chez les animaux aquatiques sont le $\mathrm{pH}$ et l'aluminium. Depuis plus de 10 ans, on sait que les populations de Salmo trutta ont fortement diminué dans les eaux contaminées de Norvège (SEVALDRUD et al., 1980). En France, l'impact des pluies acides sur la physiologie des animaux aquatiques a été très étudié par MASSABUAU (1985). Cet auteur constate en particulier, la disparition complète des truites dans les ruisseaux des Vosges où le pH est inférieur à 5 (MASSABUAU et al., 1987). Outre le pH, la toxicité des eaux acides met actuellement en cause l'aluminium qui, même à dose trace provoque la mort des Salmonidés (STOGHEIM et ROSSELAND, 1986) et plus spécifiquement celle de $S$. trutta fario dans les ruisseaux acides des Voges (PROBST et al., 1990). En ce qui concerne l'aluminium, sa toxicité chez les Poissons a été notamment étudiée par BAKER et SCHOFIELD (1982). Les études de toxicité de ce métal chez les poissons concernent en particulier le sang (WITTERS, 1986) et les branchies (YOUSON et NEVILLE, 1987).

Cependant les données obtenues par les différents auteurs, tant sur la truite (LEE et HARVEY, 1986) que sur la perche (BRUMBAUGH et KANE, 1985), montrent une grande variabilité qui serait principalement due au fait que les méthodes globales d'analyse, utilisées couramment, ne permettent pas, par 
exemple, de faire la différence entre l'aluminium adsorbé sur la surface branchiale et l'aluminium qui pourrait être intracellulaire et, dans ce cas éventuellement métabolisé.

II apparaît donc que l'aluminium soluble, dont la présence dans les eaux de rivière est liée à la dissolution de ses composés dans les sols lorsque ces derniers sont soumis aux pluies acides, est un facteur fondamental impliqué dans les problèmes de toxicité des eaux acides chez l'animal aquatique. Les organes cibles ainsi que les mécanismes cellulaires et subcellulaires de la toxicité du métal, sont actuellement bien connus chez l'homme (GALLE, 1984 ) ; ils ont déjà été étudiés en partie chez des Mollusques Bivalves et Crustacés marins (CHASSARD-BOUCHAUD et GALLE, 1986). En ce qui concerne les organismes d'eau douce et en particulier les poissons, les organes concernés par une bioaccumulation éventuelle de l'aluminium, ainsi que les mécanismes entrant en jeu, à l'échelle cellulaire et subcellulaire, ne sont encore que peu ou mal connus.

Pour tenter de comprendre les mécanismes de contamination des truites des eaux soumises aux retombées des pluies acides, contamination se traduisant essentiellement par une bioaccumulation d'aluminium, nous avons choisi d'étudier des échantilions de la truite Salmo trutta fario, récoltés dans une rivière à $\mathrm{pH}$ voisin de 5 , de la région des Vosges. Des résultats préliminaires (C. GALLE et al., 1990) obtenus sur le rein et la branchie, nous ont incités à poursuivre et à approfondir nos investigations. Dans cette nouvelle approche nous avons choisi d'analyser quelques organes et tissus : rein, foie, branchie et tube digestif. Notre but est de tenter de déterminer les points d'impact de l'aluminium et aussi de comprendre les mécanismes cellulaires et subcellulaires intervenant dans les processus de capture, de stockage et d'élimination de ce métal. En outre, nous tenterons de savoir si l'aluminium est associé à d'autres éléments minéraux. Pour cette étude, nous avons choisi d'utiliser deux méthodes de microanalyse : la spectrométrie des rayons $X$ mise au point par CASTAING (1951) et la spectrométrie de masse par émission ionique secondaire (CASTAING et SLODZIAN, 1962). Ces méthodes sont particulièrement bien adaptées à l'étude des échantillons biologiques en général (CHASSARDBOUCHAUD, 1988) et de ceux, en particulier, concernés par la pollution des systèmes aquatiques (CHASSARD-BOUCHAUD, 1987, 1991).

\section{MATÉRIEL ET MÉTHODES}

\section{Matériel biologique}

Des truites Salmo trutta fario, âgées de deux ans ont été prélevées dans la rivière Xoulces, située dans les Vosges (région de Cornimont) à l'est de la France. La récolte a été de 19 spécimens et faite au printemps au moment de la fonte des neiges. Le $\mathrm{pH}$ de l'eau était alors de 5,42 et la concentration en aluminium du milieu était de $200 \mu \mathrm{g} \mathrm{L}^{-1}$ (MASSABUAU et al., 1987). Les truites ont été disséquées dès leur capture, les différents organes prélevés puis 
traités différemment selon la technique microanalytique envisagée. 5 truites témoins ont été prélevées dans un ruisseau d'Auvergne situé à $10 \mathrm{~km}$ au sud de Clermont-Ferrand: l'eau avait un $\mathrm{pH}$ de 7,9 et elle ne contenait pas d'aluminium.

Pour la microanalyse par émission ionique secondaire, les tissus sont fixés au formol à $10 \%$, inclus dans la paraffine. Les coupes ( $5 \mu$ d'épaisseur) sont déposées sur une lame d'or poli extra pur, puis déparaffinées.

Pour la microanalyse à la sonde électronique, les échantillons sont fixés dans une solution de glutaraldehyde à $2,5 \%$ dans du tampon cacodylate $0.2 \mathrm{M}$ à $\mathrm{pH} 7,4$. La moitié des prélèvements est post-fixée dans l'osmium ; tous les échantillons sont déshydratés et inclus dans l'epon. Les coupes sont faites sur un ultramicrotome Reichert OMU2. Les coupes semi-fines ont $2 \mu \mathrm{m}$ d'épaisseur et les ultrafines $(<0,1 \mu \mathrm{m}$ d'épaisseur) sont déposées sur des grilles de cuivre. Le matériel est non osmié et non contrasté. Les grilles sont ensuite carbonées avant d'être analysées.

Le matériel destiné à être observé au microscope électronique PHILIPS EM 300, subit la doube fixation, puis les grilles sont contrastées à lacétate d'uranyle et au citrate de plomb.

\section{MÉTHODES}

Deux méthodes ont été utilisées pour cette étude : la microanalyse par sonde électronique (= microsonde de Castaing) et Ir microanalyse par émission ionique secondaire (microscope ionique). Ces $d: u x$ méthodes permettent de déterminer la composition chimique élémentaire das différents constituants cellulaires, à l'échelle structurale et ultrastructurale $\epsilon^{*}$. d'en obtenir des images de répartition. L'intérêt principal de la microscopie .. nique est sa très haute sensibilité ; en effet un élément comme l'aluminium peut être détecté dans une coupe histologique et l'image de sa répartition microscopique est obtenue, même à des concentrations extrêmement faibles, inférieures à $0,1 \mathrm{ppm}$, dans des volumes de l'ordre du micron cube, soit une sensibilité environ 1000 fois plus élevée que celle obtenue avec une microsonde électronique.

Pour un centain nombre d'éléments et en particulier pour l'aluminium, il est nécessaire de faire de la haute résolution en masse, en mode spectral, à la masse 27 pour faire la distinction entre l'ion $27 \mathrm{Al}+$ et les ions polyatomiques (principalement $\mathrm{C} 2 \mathrm{H3}=12+12+1+1+1$ ou $\mathrm{CNH}=12+14+1$ ).

Un système informatisé de numérisation et d'acquisition des images ioniques, mis au point par CAVELLIER et al. (1989) a été utilisé en association avec le microscope ionique. Son apport est fondamental dans les études relatives à l'écotoxicologie (CHASSARD-BOUCHAUD et al., 1992). 


\section{CONDITIONS D'ANALYSE}

\section{Microanalyse par spectrométrie des rayons $x$}

Les coupes ultrafines sont déposées sur grilles de cuivre, carbonées, puis observées à l'aide de la sonde CAMEBAX couplée à un microscope électronique à transmission. Les conditions d'analyse sont les suivantes : tension d'accélération de $40 \mathrm{kV}$, courant de sonde de $100 \mathrm{nA}$, diamètre de sonde de $500 \mathrm{~nm}$ environ. La détection de l'aluminium est réalisée à l'aide de spectrométres du type dispersif en longueur d'onde, équipés de cristaux PET, LIF et TAP. La raie $K \alpha$ de l'aluminium est détectée sur le PET, celle du phosphore est détectée sur le TAP.

\section{Microanalyse par émission ionlque secondaire}

Les coupes semi-fines sont déposées sur une lame d'or poli extra pur puis observées à l'aide du microscope ionique SMI 300 (CAMECA). Les conditions d'analyse sont les suivantes: bombardement par un faisceau d'ions primaires $\left(\mathrm{O}_{2}^{+}\right)$avec un courant de 500 a $800 \mathrm{nA}$; diaphragme de contraste de la lentille à immersion : $200 \mu \mathrm{m}$; résolution sur l'image : $1 \mu \mathrm{m}$ environ ; résolution en masse : 300 sur image; champ observé de $250 \mu \mathrm{m}$ de diamètre ; champ de mesure : $60 \mu \mathrm{m}$ de diamètre, tension objet : $4,5 \mathrm{kV}$.

\section{RÉSULTATS}

Les tissus ou organes suivants ont été analysés : rein, foie, branchie et tube digestif (caecum pylorique).

\section{Rein}

La figure 1 de la planche 1 présente la topographie du tissu. Les tubules rénaux sont entourés par un tissu interstitiel dans lequel on distingue en particulier des mélanocytes contenant de nombreux granules pigmentaires constitués essentiellement de mélanine.

En microscopie ionique, limage calcium permet de reconnaître la topographie du tissu (pl. 1, fig. 2) : tubules et tissu interstitiel. L'image ionique de répartition de l'aluminium (pl. 1, fig. 3) apporte de nombreuses informations. Dans l'épithélium tubulaire on observe des points particulièrement émissifs en aluminium (points blancs) disposés à l'apex des cellules et dont l'ensemble forme une couronne autour de la lumière des tubules. En outre, dans la lumière on note aussi la présence du métal. D'autres zones émissives dans le tissu interstitiel correspondent aux mélanocytes. 


\section{Planche 1}
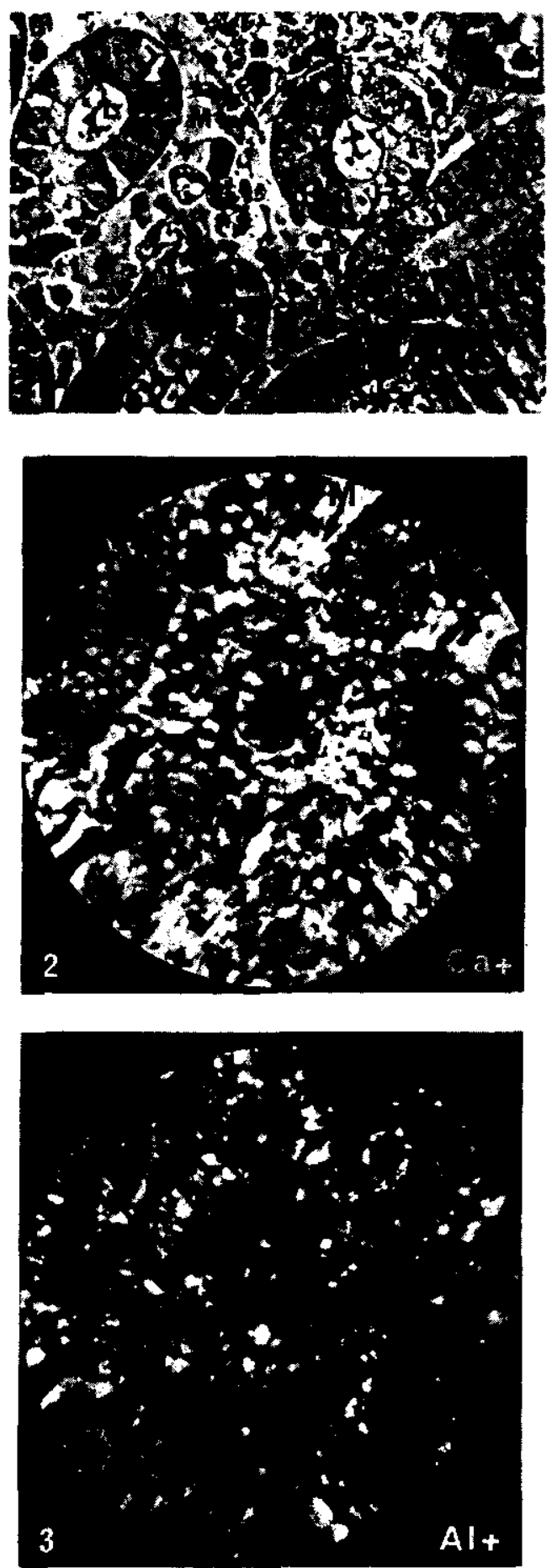

1 - Salmo trutta. Rein. Micrographie photonique d'une coupe semifine. Les tubules rénaux (T) avec leur lumière (L) sont entourés par un tissu interstitiel (ti) dans lequel on reconnaît des mélanocytes (M) contenant des granules pigmentaires (flèches). $\times 1000$.

1 -Salmo trutta. Kidney. Photon micrograph of a semithin cross section showing the tubules $(T)$ with their lumen (L) surrounded by the interstitial tissue (ti) with melanocytes (M) containing pigment granules (arrows). $\times 1000$.

2 - Salmo trutta. Rein. Image ioni que d'une coupe semi-fine montrant la topographie du tissu et la distribution du calcium dans les tubules (T) vus en coupes transversales et entourés du tissu interstitiel (ti) contenant des mélanocytes (M). $\times 800$.

2 - Salmo trutta. Kidney. ${ }^{40} \mathrm{Ca}^{+}$ion image of a semithin section showing the tissue topography and the calcium emission from tubules $(T)$ surrounded by the interstitial tissue (ti) with melanocytes $(M)$. $\times 800$.

3 - Salmo trutta. Rein. Image ionique obtenue à partir de la même coupe que celle de la figure 2 montrant une forte émission d'aluminium par les tubules, essentiellement au niveau de points correspondant à des lysosomes disposés en couronne dans la zone apicale des cellules epitheliales (petites flecches). L'aluminium est aussi détecté dans la lumière (L) des tubules. Dans le tissu interstitiel (ti) on note une forte émissivité d'aluminium au niveau des granules pigmentaires des mélanocytes (M). $\times 800$.

3 - Salmo trutta. Kidney. ${ }^{27} \mathrm{Al}+$ ion image obtained from the same area as figure 2, showing aluminium emission from tubule lysosomes with a ring-shaped localization along the apical border of the epithelial cells (small arrows). Aluminium emission is also observed from the tubule lumen ( $L$ ) and from the pigment granules of the melanocytes $(M)$ located within the interstitial tissue (ti). $x 800$ 
Les coupes ultrafines de ce tissu rénal sont analysées à la sonde Camebax. Dans les cellules épithéliales tubulaires on reconnaît de nombreux lysosomes ( $p l .2$ ) en situation apicale et qui renferment de l'aluminium associé à du phosphore: ils correspondent aux points très émissifs en aluminium, disposés en couronne sur les images ioniques. Les granules pigmentaires des mélanocytes contiennent de l'aluminium associé à du phosphore, comme le montrent les raies $X$ obtenues sur ces organites ( $p l .3$ ).

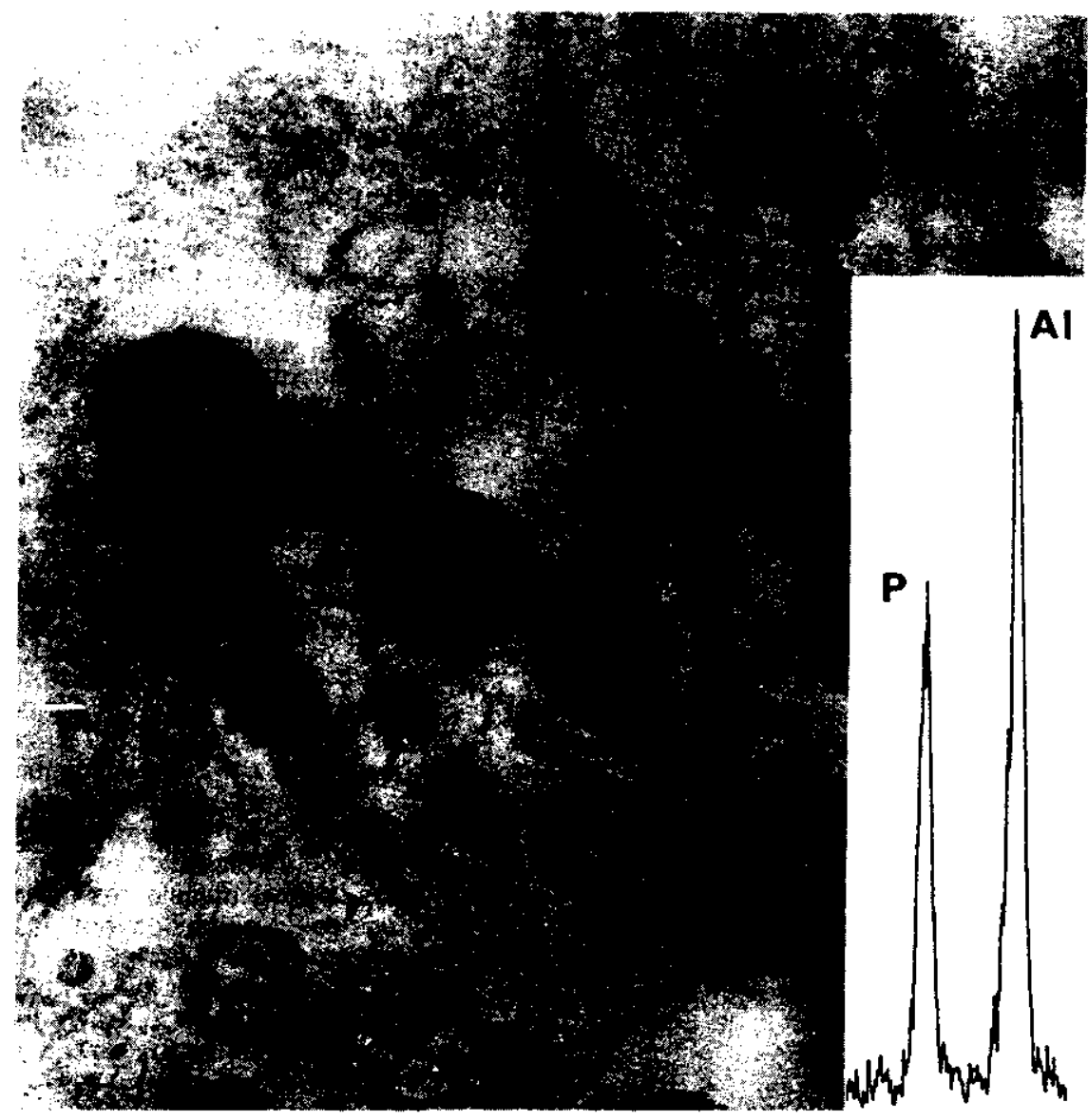

Planche 2 Salmo trutta. Rein. Matériel non osmié et non contrasté. Micrographie électronique montrant des lysosomes (L) localisés dans la zone apicale des cellules épithéliales tubulaires et contenant de laluminium associé à du phosphore. A droite, les spectres de rayons $X$ d'Al et $P$ obtenus sur ces lysosomes à l'aide de la microsonde électronique CAMEBAX. X 40000 .

Salmo trutta. Kidney. Non osmicated and unstained material. Electron micrograph showing lysosomes (L) located along the apical border of the tubular epithelial cells and containing aluminium associated with phosphorus. Inset : $X$ ray emission spectra of $A /$ and $P$, obtained from these lysosomes, using the CAMEBAX electron microprobe. $X 40000$. 


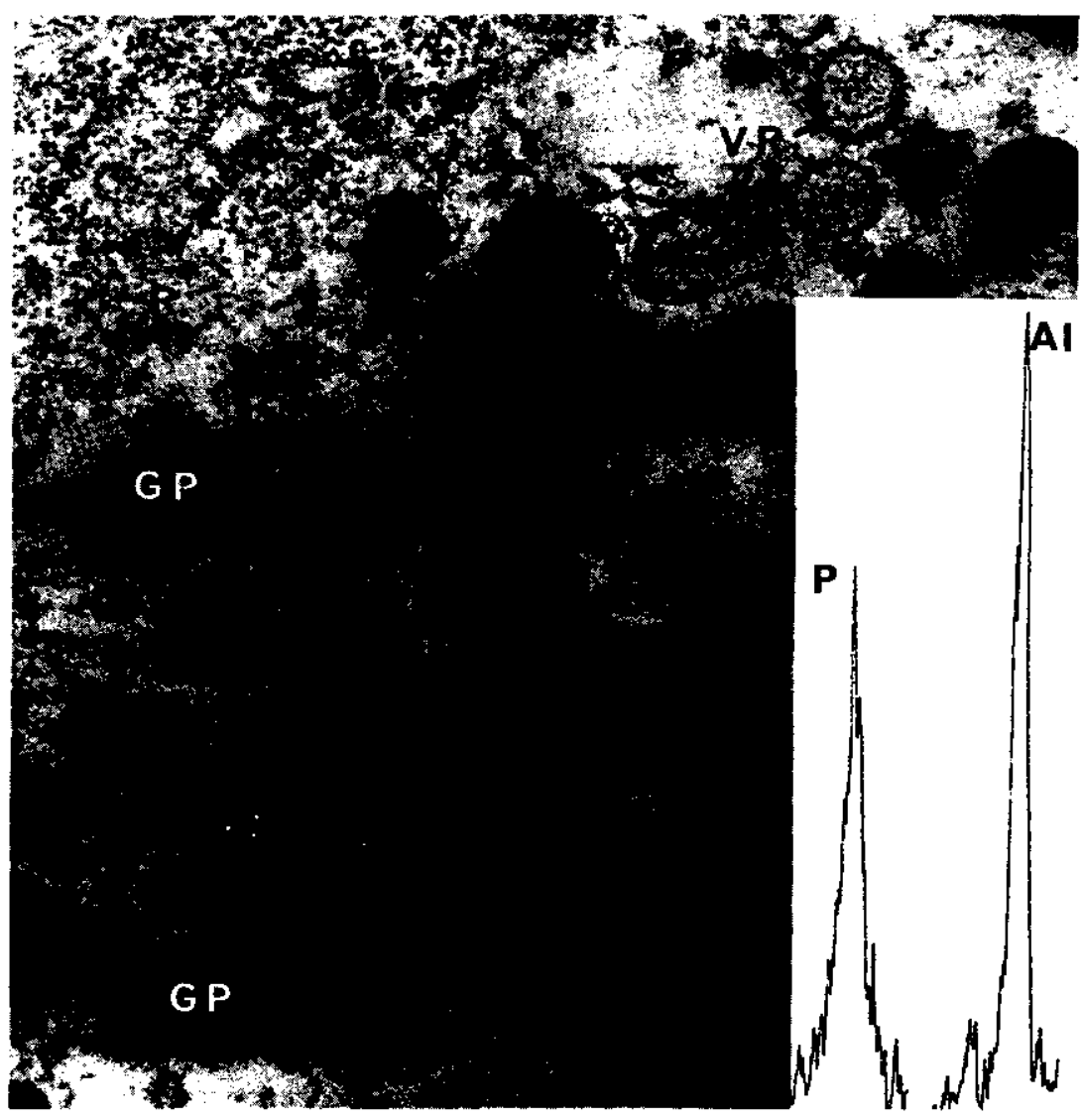

Planche 3 Salmo trutta. Rein. Micrographie électronique montrant les granules pigmentaires (GP) d'un mélanocyte qui contiennent de l'aluminium associé à du phosphore. $M$ : mitochondrie, $R$ : ribosomes, VR : vésicule recouverte. A droite : les spectres de rayons $X$ d'Al et $P$ obtenus sur ces granules pigmentaires à l'aide de la microsonde électronique CAMEBAX. X 40000 .

Salmo trutta. Kidney. Electron micrograph showing the pigment granules (GP) of a melanocyte containing aluminium associated with phosphorus. $M$ : mitochondria, $R$ : ribosome, VR: coated vesicle. Inset : $X$-ray emission spectra of $A l$ and $P$ obtained from these pigment granules using the CAMEBAX electron microprobe. $\times 40000$.

Il apparaît ainsi que, dans le tissu rénal, les lysosomes de l'épithélium tubulaire et les granules pigmentaires des mélanocytes du tissu interstitiel sont les deux sites électifs de bioaccumulation du métal. Aucun signal significatif indiquant la présence d'aluminium n'a été obtenu à partir des autres organites de ce tissu des truites des Vosges, ni de l'ensemble de ce tissu des truites témoins. 


\section{Foie}

Obtenue par microscopie ionique, l'image calcium (pl. 4, fig. 1) permet de reconnaître la topographie de la coupe où l'on distingue outre le tissu hépatique, un vaisseau en coupe longitudinale, contenant des globules sanguins. L'image aluminium (pl. 4, fig. 2) fait apparaître de nombreux points blancs correspondant à la présence du métal dans les cellules hépatiques ainsi que dans quelques globules sanguins.

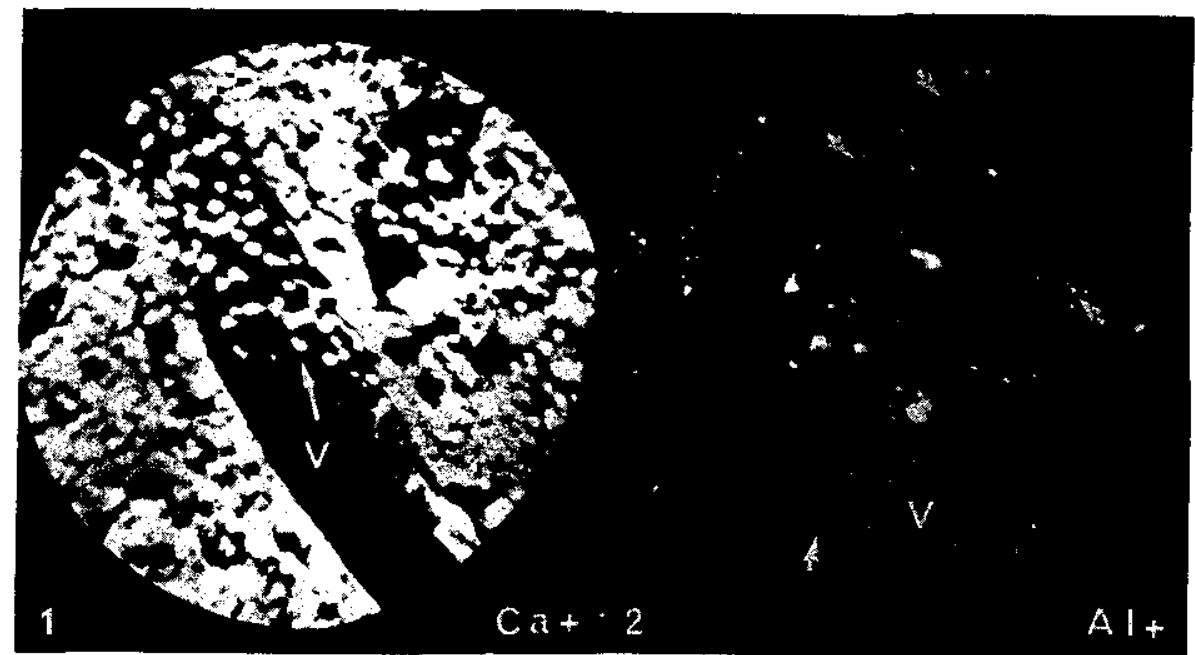

Planche 41 - Salmo trutta. Foie. Image ionique d'une coupe semi-fine montrant la topographie du tissu et la distribution du calcium dans les cellules hépatiques ainsi que dans les globules du vaisseau sanguin (V). $\times 800$. 1 -Salmo trutta. Liver. ${ }^{40} \mathrm{Ca}^{+}$ion image of a semithin section showing the tissue topography and the calcium emission from the liver cells and from the blood cells located within the vessel (V). $\times 800$.

2 - Salmo trutta. Foie. Image ionique obtenue à partir de la même coupe que celle de la figure 1 , montrant des points riches en aluminium dans le tissu hépatique (flèches) ainsi que dans certains globules du vaisseau sanguin (V). $\times 800$.

2 - Salmo trutta. Liver. ${ }^{27} \mathrm{Al}+$ ion image obtained from the same area as figure 1 showing aluminium emission from small points in the liver tissue (arrows) and from some blood cells of the vessel (V). X 800 .

L'examen des coupes ultrafines de foie, à l'aide de la micro sonde électronique, permet une détection d'aluminium et de phosphore dans les lysosomes des cellules hépatiques.

Le foie des truites témoins, analysé par les deux techniques, à l'échelle structurale et ultrastructurale, n'a révélé aucune présence significative d'aluminium. 


\section{Branchie}

Cet organe a été examiné en analyse ionique. L'image calcium ( $p$ ). 5 , fig. 1) représente la topographie de la coupe où lon reconnaît la lamelle principale portant des lamelles secondaires... L'image aluminium ( $p l .5$, fig. 2) obtenue sur la même coupe que celle de la figure 1, met en évidence une forte accumulation de particules riches en métal, au niveau des lamelles secondaires qui sont en contact direct avec le milieu extérieur.

En utilisant la microsonde électronique, on démontre que les deux sites cellutaires de bioaccumulation de l'aluminium, toujours associé à du phosphore, sont les mêmes que ceux mis en évidence dans le rein. Ce sont les granules pigmentaires et les lysosomes qui sont particulièrement abondants dans la région apicale des cellules épithéliales.

La branchie des truites témoins, analysée par les deux techniques à l'échelle structurale, n'a révélé, comme dans le cas du foie, aucune présence significative d'aluminium.

\section{Tube digestif : caecum pylorique}

Nous avons examiné ces éléments du tube digestif des téléostéens, qui sont des expansions étroites et allongées de l'intestin, présentant de nombreux replis qui augmentent considérablement la surface de digestion et d'absorption.

L'image ionique de la répartition du calcium (pl. 6, fig. 1) permet d'interpréter la topographie de la coupe transversale d'un caecum. Sous l'assise épithéliale (E) se trouve une couche de muscles circulaires (MC), puis une couche de muscles longitudinaux $(\mathrm{ML})$. Traversant ces trois couches cellulaires, on observe un volumineux dépôt mesurant environ 120 sur $100 \mu \mathrm{m}$, très calcifié. L'image ionique de la répartition de l'aluminium (pl. 6, fig. 2) démontre que cette inclusion est particulièrement riche en aluminium. D'autres points faiblement émissifs sont visibles dans la coupe, particulièrement dans la lumière où se trouvent quelques particules minérales. témoins.

De tels dépôts d'aluminium n'ont pas été observés chez les animaux

\section{DISCUSSION}

Pour tenter de déterminer une bioaccumulation éventuelle d'aluminium chez la truite Salmo trutta fario, de la localiser avec précision à l'échelle structurale et ultrastructurale et de comprendre les mécanismes cellulaires qui la régissent, nous avons donc, en première approche analysé quelques tissus et organes. La branchie qui est le principal organe d'échanges avec le milieu extérieur et le rein qui est par définition l'organe excréteur, devaient être 

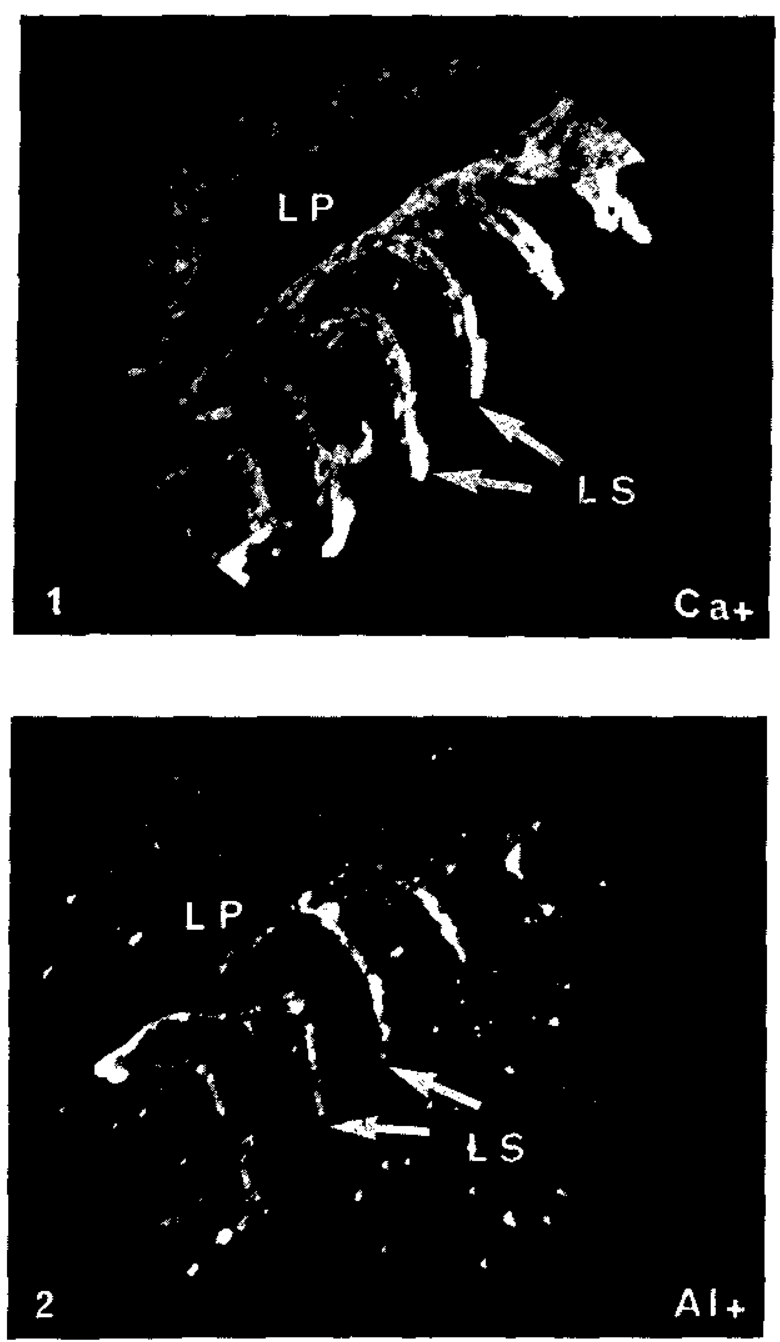

Planche 51 - Salmo trutta. Branchie. Image ionique d'une coupe semi-fine montrant la topographie du tissu et la distribution du calcium dans les lamelles secondaires (LS) et dans la lamelle primaire (LP). X 800 .

1 - Salmo trutta. Gill. ${ }^{\circ} \mathrm{Ca}^{+}$ion image of a semithin section showing the calcium emission from the secondary lamellae (LS) and from the primary lamella (LP). $\times 800$.

2 - Salmo trutta. Branchie. Image ionique obtenue à partir de la même coupe que celle de la figure 1 montrant une émission d'aluminium, forte au niveau des lamelles secondaires (LS), plus faible au niveau de la lamelle primaire (LP). $\times 800$.

2 - Salmo trutta. Gill. ${ }^{27} \mathrm{Al}^{+}$ion image obtained from the same area as figure 1 showing a high aluminium emission from the secondary lamellae (LS) and a weak one from the primary lamella (LP). $X 800$. 

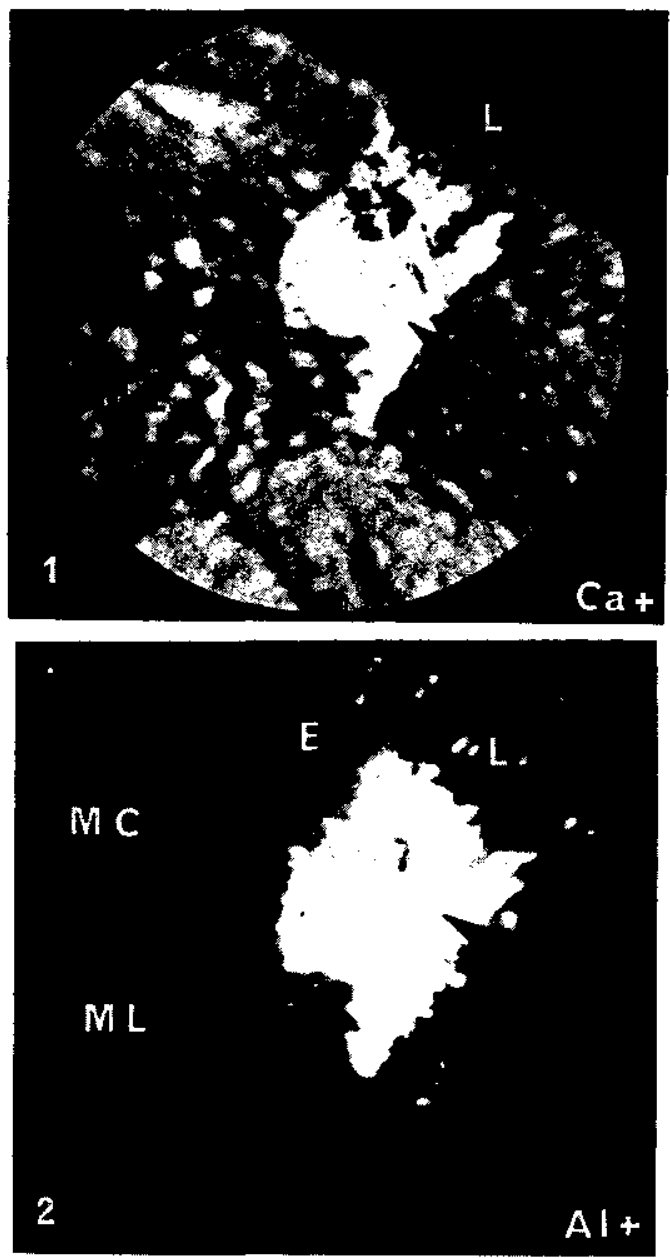

Planche 61 - Salmo trutta. Caecum pylorique. Image ionique d'une coupe transversale semi-fine montrant la topographie du tissu et la répartition du calcium. E : épithélium, $L$ : lumière, $M C$ : muscies circulaires, $M L$ : muscles longitudinaux. La flèche indique la localisation d'un énorme dépôt très calcifié. $\times 800$.

1 - Salmo trutta. Pyloric caecum. ${ }^{40} \mathrm{Ca}^{+}$ion image of a semithin cross section showing the tissue topography. $E$ : epithelium, $L$ : lumen, $M C$ : circular muscles, ML: longitudinal muscles. Arrow indicates a large calcium deposit. $\times 800$.

2 - Salmo trutta. Caecum pylorique. Image ionique d'aluminium obtenue à partir de la me̊me coupe que celle de la figure 1. L'énorme dépôt (flèche) montre une très forte émissivité en aluminium. La lumière (L) en contient quelques particules. $E$ : épithélium, MC: muscles circulaires, $M L$ : muscles longitudinaux. $X 800$.

2 - Salmo trutta. Pyloric caecum. ${ }^{27} \mathrm{~A} /+$ ion image obtained from the same area as figure 1. The large deposit (arrow) shows a high aluminium emission. Small particles of the metal are to be seen in the lumen (L). E: epithelium, MC : circular muscles, $M L$ : longitudinal muscles. $X 800$. 
examinés en premier lieu. Nous nous sommes aussi intéressés à une partie de l'appareil digestif. Le foie qui est le site de bioaccumulation des réserves métaboliques est aussi un organe très sensible à beaucoup de causes d'intoxications ou de troubles pathologiques divers. Les caecum pyloriques ont aussi retenu notre attention, étant donnée l'immense surface d'échange qu'ils représentent dans les processus de digestion et d'absorption.

Il convient de souligner que la détermination rigoureuse de l'aluminium dans les échantillons biologiques est difficile à réaliser. En effet, nombreux sont les problèmes causés soit par la contamination, soit par une perte du métal lors des différentes étapes de récolte puis de traitement du matériel biologique. Pour effectuer des analyses globales, les auteurs font généralement appel à l'activation neutronique et à la spectroscopie par absorption atomique au four graphite (KRATOCHVIL et al., 1987). Mais ces auteurs sont conscients de la possibilité de nombreux artefacts, en particulier de ceux dus à la présence de silicoaluminates, impossibles à éliminer étant donnée leur présence généralisée dans tous les milieux. En outre ces analyses globales ne renseignent pas, comme nous l'avons souligné, sur l'éventuelle incorporation tissulaire et cellulaire du métal.

Grâce à nos techniques de microanalyse, nous avons pu démontrer que l'aluminium que nous avons détecté n'est pas associé à du silicium. Ces résultats excluent ainsi totalement la possibilité de la présence de silicoaluminates, provenant d'apports d'origine exogène et qui seraient à l'origine de graves artefacts.

Utilisant des techniques d'analyse globale, BRUMBAUGH et KANE (1985) constatent dans les branchies de Micropterus dolomieui des concentrations importantes d'aluminium, concentrations cependant très variables. If apparaît que ces résultats peuvent s'expliquer par le fait que les méthodes employées ne permettent pas de faire la distinction entre l'aluminium adsorbé en surface par la couche de mucus et l'aluminium intracellulaire métabolisé.

L'utilisation de la microsonde électronique permet à YOUSON et NEVILLE (1987) de relater la présence, chez Salmo gairdneri, de " particules " riches en aluminium, situées sur la surface épithéliale branchiale et de « vésicules et corps denses cytoplasmiques". Par rapport aux résultats précités concernant la branchie, nos résultats apportent grâce aux données microanalytiques obtenues, tant à l'échelle structurale, qu'à l'échelle ultrastructurale, des informations qui précisent les types d'organites concernés par les processus de bioaccumulation de l'aluminium et la nature exacte des "vésicules " intervenant dans la cellule, pour stocker le métal.

Le lysosome est le site bien-connu de concentration de nombreux éléments minéraux stables ou radioactifs (CHASSARD-BOUCHAUD, 1985), chez les organismes aquatiques vivant dans des milieux plus ou moins pollués; ces éléments sont généralement associés à du phosphore, et insolubilisés sous forme de phosphate, réaction due à l'activité phosphatasique acide propre au lysosome (BERRY et al., 1982 ; CHASSARD-BOUCHAUD, 1983). Nos résultats, obtenus chez la truite, sont à rapprocher de ceux obtenus chez des Mollusques Bivalves et chez des Crustacés Décapodes d'origines très diverses (Europe, Amérique, Asie). Chez ces organismes, l'organe cible 
principal de bioaccumulation est la glande digestive, l'organite cible étant, comme dans le cas des truites, le lysosome dans lequel l'aluminium est précipité sous forme de phosphate insoluble (CHASSARD-BOUCHAUD et GALLE, 1986).

L'autre organite cible de concentration de l'aluminium chez la truite est le granule pigmentaire. En ce qui concerne la capacité de certains types de pigments de pouvoir piéger des métaux, les résultats présents sont à rapprocher de résultats antérieurs qui nous avaient permis de démontrer, par microanalyse, que les mélanines et ommochromes des Crustacés sont associées à différents éléments minéraux (CHASSARD-BOUCHAUD, 1972). Nous avions aussi, par les mêmes méthodes d'analyse, détecté dans les granules pigmentaires des mélanophores du saumon, plusieurs métaux et en particulier de l'aluminium (CHASSARD-BOUCHAUD, 1982). Les granules pigmentaires de l'épithélium branchial et ceux du tissu interstitiel rénal, constituent ainsi des sites de bioaccumulation de l'aluminium. Dans le rein, le stockage puis l'excrétion de l'aluminium sont assurés par les lysosomes des cellules tubulaires qui concentrent le métal, puis le rejettent, par exocytose, dans la lumière des tubules où le métal est notoirement abondant. Rappelons aussi la présence d'aluminium dans la lumière des caecums pyloriques.

Outre une localisation intracellulaire de l'aluminium, nous avons mis en évidence la présence d'importants et volumineux dépôts du métal qui affectent trois couches cellulaires : une assise épithéliale et les deux couches musculaires circulaire et longitudinale; dans cette zone, les tissus sont totalement détruits. II ne s'agit pas d'un phénomène isolé. En effet, des dépôts d'aluminium de taille comparable ont été observés dans d'autres tissus de ces truites ainsi que dans des brêmes vivant en milieu acide et enrichi en aluminium. Ces dépôts se situent dans des organes incapables d'excréter et ils détruisent les tissus. Ces derniers résultats font actuellement l'objet d'une publication en préparation.

On connaît bien chez l'homme (GALLE, 1984), grâce à l'utilisation de techniques microanalytiques, qui sont les mêmes que les nôtres, les organes cibles et les mécanismes cellulaires impliqués dans les intoxications par l'aluminium. Ces observations ont été faites sur des biopsies de différents organes effectuées sur des malades décédés après traitement par dialyse avec une eau riche en aluminium (LAPRESLE et al., 1975), dialyse rendue nécessaire à cause d'insuffisance rénale. Ces résultats démontraient l'existence de volumineux dépôts de phosphate d'aluminium qui engendraient localement, dans des tissus incapables d'excrétion, des destructions cellulaires plus ou moins étendues. Nos résultats obtenus sur la truite sont tout à fait comparables. De tels dépôts de métaux, non contenus dans des organites limités par une membrane, bien qu'étant en situation intracellulaire, sont rarement observés chez les animaux aquatiques. Signalons, cependant, la mise en évidence de microdépôts de chrome dans le tissu musculaire d'invertébrés marins (CHASSARD-BOUCHAUD et al., 1989).

Dans le présent travail, l'évaluation quantitative de la concentration de l'aluminium n'a pas été abordée, étant donné que celle-ci ne constituait nullement le but de notre étude qui est une approche des mécanismes cellulaires et subcellulaires de la bioaccumulation du métal. Cet aspect 
quantitatif est actuellement en cours d'étude sur les différents organes de la truite grâce à des méthodes d'analyse globale.

En conclusion, l'aluminium devenu biodisponible sous forme soluble en milieu acide, dans l'eau des rivières des Vosges, peut être concentré par la truite, dans certains de ses tissus et avec différentes modalités entraînant une bioaccumulation soit ponctuelle et intracellulaire, soit massive et extracellulaire. Le premier processus est un processus classique de bioconcentration et d'insolubilisation du métal sous forme de phosphate, dans les lysosomes où intervient une activité enzymatique phosphatasique acide. Le deuxième processus de piégeage intracellulaire est assuré par les pigments des granules contenus dans les mélanosomes de différents tissus. Ces deux mécanismes contribuent à isoler le métal du milieu cellulaire et à atténuer sa toxicité. Le troisième processus observé, de grande amplitude, entraîne la formation de volumineux dépôts métalliques pouvant affecter plusieurs couches cellulaires d'un tissu ou d'un organe et provoquer leur destruction plus ou moins étendue.

Nos travaux se poursuivent actuellement sur d'autres organes de ces mêmes truites pour savoir s'ils sont aussi atteints par ces phénomènes de bioconcentration d'aluminium et selon quelles modalités.

Rappelons enfin les travaux de PROBST et al. (1990) selon lesquels les basses valeurs de pH qui entraînent une mise en solution de laluminium, sont les facteurs clés qui déterminent la mortalité des truites, à partir de $200 \mu \mathrm{g}^{\mathrm{L}-1}$ d'aluminium dissous dans les rivières des Vosges. Ces conditions de milieux étant précisément celles des ruisseaux où ont été prélevés les poissons que nous avons analysés, on peut émettre l'hypothèse selon laquelle les modalités de bioconcentration de l'aluminium que nous avons décrites, pourraient être en grande partie responsables de la disparition des truites dans les cours d'eau soumis aux retombées des pluies acides.

\section{REMERCIEMENTS}

Les auteurs remercient J.C. MASSABUAU qui a récolté une partie du matériel analysé, dans les ruisseaux acides des Vosges. Ce travail a été réalisé avec la collaboration technique de F. KLEINBAUER qui a assuré la préparation des échantillons pour les observations et microanalyses à l'échelle structurale et ultrastructurale et avec celle de F. ESCAIG et P. BOUMATI qui ont collaboré aux microanalyses.

La microscopie électronique a été effectuée au Laboratoire de Microscopie Electronique de l'Université P. et M. Curie, 105, boulevard Raspail, F 75006 Paris, France. 


\section{RÉFÉRENCES BIBLIOGRAPHIQUES}

BAKER J.P., SCHOFIELD C.L., 1982. Aluminum toxicity to fish in acidic waters. Water. air and soil polkution, 18, 289-309.

BERRY J.P., HOURDRY J., STERNBERG M., GALLE P., 1982. Aluminium phosphate visualisation of acid phosphatase activity : a biochemical and $\mathrm{X}$-ray microanalysis study. J. Histochem. Cytochem, 30, 86-90.

BONNEAU M., LANDMAN G., 1988. Le dépérissement des foréts en Europe. $L$ a Recherche, 205 (19), 1542-1556.

BRUMBAUGH W.G., KANE D.A., 1985, Variability of aluminum concentrations in organs and whole bodies of small mouth bass (Micropterus dolomieui). Environ. Sci. Technol., 19, 828-831.

CASTAING R., 1951. Application des sondes electroniques à une méthode d'analyse ponctuelle et cristallographique. These de Doctorat d'Etat, Onera ed., Paris.

CASTAING R., SLODZIAN G., 1962. Microanalyse par émission ionique secondaire. J. de Microscopie, 1, 395-410.

CAVELLIER J.F., BERRY J.P., ESCAIG F., BOUMATI P., GAUME P., 1989. Processing of secondary ion microscope images : an example of application to the thyroid. $J$. of Microsc., 154, 31-38.

CHASSARD-BOUCHAUD C., 1972. Analyse chimique des chromatophores de Crangon crangon (Linné) Crustacé Décapode, par spectrographie des rayons $X$. Differences quantitatives de composition en éléments $\mathrm{Ca}, \mathrm{P}, \mathrm{S}$ et $\mathrm{Mg}$ au cours du cyle d'intermue. C. R. Acad. Sc. Paris, 274, série D, 25112514.

CHASSARD-BOUCHAUD C., 1982. Programme coordonné d'études sur les nécroses des animaux marins. Ulcérations des poissons et mammifères marins péchés dans les eaux cótières françaises. Contrat CNEXO n० 79/2126.

CHASSARD-BOUCHAUD C., 1983. Cellular and subcellular localization of uranium in the crab Carcinus maenas : a microanalytical study. Mar. Poll. Bull., 14 (4), 133-136.

CHASSARD-BOUCHAUD C., 1985. Bioaccumulation de métaux stables et radioactifs par les organismes benthiques de la Baie de Seine. Aspects structuraux, ultrastructuraux et microanalytiques. Cah. Biol. Mar. 26, 63-86.

CHASSARD-BOUCHAUD C., 1987. IOn $\mathrm{mi}$ croscope and microprobes in marine poltution research. Analytica Chimica Acta, 195. 307-315.

CHASSARD-BOUCHAUD C., 1991. MicroanaIytical techniques in toxicological investigations in ECOTOXICOLOGY AND THE MARINE ENVIRONMENT. Edited by P.D. Abel and V. Axiak, Ellis Horwood, New York, p. 176-200.

CHASSARD-BOUCHAUD C., BOUTIN J.F. HALLEGOT P., GALLE P., 1989. Chromium uptake, distribution and loss in the mussel, Mytilus edulis: a structurai, ultrastnuctural and microanalytical study. Dis Aquat. Org., 7, 117-136.

CHASSARD-BOUCHAUD C., ESCAIG F., BOUMATI P., GALLE C., 1992. Microanaly. sis and image processing of stable and radioactive elements in ecotoxicology. Current developments using SIMS microscope and electron microprobe. Biol. Cell., 74, 59-74.

CHASSARD-BOUCHAUD C., GALLE P., 1986. Bioaccumulation d'aluminium par les organismes marins. Mise en évidence par microscopie corpusculaire analytique. $C . R$. Acad. Sc. Panis, 302, série III (2), 55-61.

GALLE C., CHASSARD-BOUCHAUD C., MASSABUAU J.C., ESCAIG F., BOUMATI P., BOURGES M., PEPIN D., 1990. Localisation subcellutaire de l'aluminium véhiculé par les pluies acides dans les reins et branchies de truite des Vosges. Données préliminaires. C. R. Acad. Sc. Paris, 311 , série III, 301-307.

GALLE P., 1984. Physiopathologie cellulaire de l'aluminium. Aluminium et Insuftisance rénale, Paris, Ed. Gambro, 119-129.

KRATOCHVIL B., MOTKOSKY N., DUKE J.M., Ng. D., 1987. Determination of trace aluminum concentration and homogeneity in biological reference material TORT-1 by instrumental neutron activation analysis and graphite furnace atomic absorption spectroscopy. Can. J. Chem., 65, 10471050. 
LAPRESLE J., DUCKETT S., GALLE P., 1975. Documents cliniques, anatomiques et biophysiques dans une encéphalopathie avec présence de dépóts d'aluminium. C. R. Soc. Biol. Paris, 169, 282-285.

LEE C., HARVEY H., 1986. Localization of alu minum in tissues of fish. Water, air and soil pollution, 30, 649-655.

MASSABUAU J.C., 1985. Pluies acides et physiologie des animaux aquatiques. Bull. Soc. Ecophysiol., 10 (2), 59-74.

MASSABUAU J.C., FRITZ B., BURTIN, 1987. Mise en évidence de ruisseaux acides ( $\mathrm{pH} \leq 5)$ dans les Vosges. C. R. Acad. Sci. Paris, 305, série III, 121-124.

MOHMEN M., 1988. Le danger des pluies acides. Pour la Science, 132, 54-63.

OVERREIN L., SEIP H., TOLLAN A., 1981. Acid precipitation. Effects on forest and fish. Final report of the SNSF Project 1972-1980.

PROBST A. MASSABUAU J.C., PROBST J.L., FRITZ B., 1990. Acidification des eaux de surface sous l'influence des précipitations acides: róle de la végétation et du substratum, conséquences pour les populations de truites. Le cas des ruisseaux des Vosges. C. R. Acad. Sci. Paris, 311 , série II, 405-411.

SEVALDRUD I.H., MUNIZ I.P., KALVENES S., 1980. Loss of fish populations in Southern Norway. Dynamics and magnitude of the problem. In Drablos D. and Trollan A. Eco logical impact of acid precipitation, 350351.

SKOGHEIM O.K., ROSSELAND B.O., 1986 Mortatity of smolt of atlantic saimon Salmo salar $\mathrm{L}$. at low tevels of aluminum in acidic softwater. Bull. Environ. Contam. Toxicol., 37, 258-265.

WITTERS H.E., 1986. Acute acid exposure of rainbow trout Salmo gairdneri Richardson) effects of aluminum and calcium on ion balance and haematologgy. Aquatic Toxicol., 8, 197-210.

YOUSON J.H., NEVILLE C.M., 1987. Deposition of aluminum in the gill epithelium of rainbow trout (Salmo gairdneri Richardson) subjected to sublethal concentrations of the metal. Can J. Zool., $65,647-656$. 